\title{
Thermal trimming and tuning of hydrogenated amorphous silicon nano-photonic devices
}

\author{
Shankar Kumar Selvaraja,* Wim Bogaerts, and Dries VanThourhout \\ Ghent University-imec, Photonic research group, \\ Department of Information Technology, Ghent 9000, Belgium. \\ Marc Schaekers \\ imec, Leuven, Belgium.
}

(Dated: July 27, 2010)

\begin{abstract}
Deposited silicon and in particular hydrogenated amorphous silicon forms an attractive alternative platform for realizing compact photonic integrated circuits. In this paper we report on trimming (towards lower wavelengths) and tuning (towards higher wavelengths) of photonic devices through a suitable thermal treatment. The former is achieved by a material density change, the latter through the thermo-optic effect. By using fourier transform infrared spectroscopy, a change in the hydrogen content is identified as the source of the density change. A total wavelenght tuning range of $24.6 \mathrm{~nm}$ is achievable, which can be used for compensating fabrication imperfections.
\end{abstract}


The use of deposited silicon layers instead of crystalline silicon-on-insulator wafers forms an attractive alternative for realizing compact integrated photonic devices ${ }^{1,2}$. Recently, we have demonstrated low-loss photonics wires and wavelength selective devices fabricated in hydrogenated amorphous silicon $(\mathrm{a}-\mathrm{Si}: \mathrm{H})^{1}$. Plasma enhanced chemical vapour deposition (PECVD) with $\mathrm{SiH}_{4}$ as the precursor is widely used for achieving a low-loss guiding layer for photonic applications. Because of the reduced process temperature, PECVD deposition is a promising process for integrating photonics ciruits on top of prefabricated electronic circuits, since this imposes strong constraints on the processing and operating temperature $(<$ $400^{\circ} \mathrm{C}$ ). In addition, the PECVD process allows for dangling bond reduction during the deposition process through hydrogen passivation of the film. The properties of a-Si:H, such as the refractive index and crystallinity are strongly influenced by the amount of hydrogen $(\mathrm{H})$ incorporated in the film, and can be controlled by process parameters, such as the substrate temperature, the rf power and the gas flow rate. When the film is in thermodynamic equilibrium, the $\mathrm{H}$ in an a-Si:H network manifests itself in different configurations $\left(\mathrm{SiH}_{x=1-3}\right)$ and bond energies. If the equilibrium in the film is disturbed optically ${ }^{3,4}$ or thermally ${ }^{5-7}$, bond breaking and reorganization of the network occurs ${ }^{8}$. This results in hydrogen desorption and crystallization. Despite considerable material development, there are no reports on the thermal properties of a-Si:H used for photonic integrated circuits.

In this paper, we present the effects of a thermal treatment on propagation loss and refractive index change of photonic devices fabricated in a-Si:H. The devices were annealed at high temperatures $\left(150-600^{\circ} \mathrm{C}\right)$ followed by optical characterization at room temperature. The material compositional changes were studied using Fourier transform infra-red spectroscopy (FTIR) and compared with the device characteristics. In addition, the devices were characterized at elevated temperatures to measure the thermo-optic (TO) effect of a-Si:H. Finally, 
we present an approach to use the thermal properties of a-Si:H for compensating fabrications imperfection of the devices ${ }^{9}$.

The thermal effect of a-Si:H was studied by using two types of photonic devices: MachZehnder interferometers (MZI) and long spiral waveguides (Fig. 1). The Mach-Zehnder interferometer is used to determine refractive index changes by measuring the spectral response of the device. The spiral waveguides are used to characterize material absorption losses, which can be estimated from the waveguide propagation loss. The $1 \times 1$ MZI was composed of two connected Y-splitters with a delay length $(\delta \mathrm{L})$ of $50 \mu \mathrm{m}$ in one of the two arms (Fig. 1a) . The spiral photonic wires of varying length $(0.5$ to $40 \mathrm{~cm})$ were realized using straight and bend waveguides. Large bending radii $(20 \mu m)$ were used to avoid any bend loss. The cross section of the photonic wire was $450 \times 220 \mathrm{~nm}$, resulting in single mode waveguides.

We fabricated the devices on a $200 \mathrm{~mm}$ wafer with $220 \mathrm{~nm}$ of low-loss a-Si:H $\mathrm{H}^{1}$ as the waveguiding layer. The waveguide layer was deposited using a PECVD process on top of 2000nm surface polished high-density plasma silicon dioxide. After layer deposition, the photonic devices were fabricated using $193 \mathrm{~nm}$ optical lithography and dry etching ${ }^{10}$. Next the wafer was diced into individual samples. Then the devices in the different samples were optically characterized to obtain the reference spectral response and propagation loss. The optical characterization was done by injecting TE polarized broadband (1500-1600nm) light from a superluminicent light emitting diode (SLED) and measuring the output through an optical spectrum analyser. Identical grating fiber-chip couplers were used for in and out coupling of light ${ }^{11}$. After reference measurements, each sample was annealed at different temperatures ranging from 100 to $600^{\circ} \mathrm{C}$ for 30 minutes in a nitrogen ambiance. To avoid thermal shock, the samples were loaded and unloaded at cleanroom temperature, while 
the raise and fall temperature was set at $10^{\circ} \mathrm{C} / \mathrm{min}$. Finally, the annealed samples were optically characterized again at room temperature. In addition, unannealed samples were characterized at elevated temperatures to measure the thermo-optic effect.

As shown in Fig. 2, we observe a blue shift in the spectral response of the MZI's after annealing. The observed spectral shift is a consequence of a reduction in the effective refractive index $\left(N_{\text {eff }}\right)$ of the photonic wire. Through numerical simulations using scattering matrix method, we have found that $1 \mathrm{~nm}$ wavelength shift corresponds to a change of $2.9 \mathrm{e}-3$ in the effective refractive index of the zeroth order guided mode (Fig. 5).

The measured wavelength shift follows a linear trend beyond a critical temperature $\left(\mathrm{T}_{c}\right)$ of $200^{\circ} \mathrm{C}$ (Fig. 3) reaching a maximum shift of $-6.6 \mathrm{~nm}$ at $400^{\circ} \mathrm{C}$. However, below the critical temperature $\mathrm{T}_{c}$ no wavelength response is observed. Due to the strongly increasing propagation loss, we were unable to measure samples annealed above $400^{\circ} \mathrm{C}$. However, for temperatures below $400^{\circ} \mathrm{C}$ the loss did not vary beyond $0.2 \mathrm{~dB} / \mathrm{cm}($ Fig. 4$)$.

The change in the spectral response and the propagation loss can be directly related to permanent change in the material property of a-Si:H after annealing. The amount of $\mathrm{H}$ in a silicon network affects the properties of the a-Si:H film, including the film density. It is well known that at an elevated temperature the mobility of $\mathrm{H}$ in the film increases, resulting in desorption of $\mathrm{H}$ from the $\mathrm{Si}$ network ${ }^{5}$. The two main consequences of $\mathrm{H}$ desorption are decrease in refractive index and creation of Si dangling bonds. The three important FTIR phonon mode bands related to the different Si-H bond configurations are located at 640, 2000 and $2100 \mathrm{~cm}^{-1}$. Figure 6 and 7 show the integrated intensity and the absorption spectra for Si-H monohydride at 2000 and $640 \mathrm{~cm}^{-1}$. It is obvious from these figures that the intensity of the 2000 and $640 \mathrm{~cm}^{-1}$ peaks starts to decrease beyond the critical temperature $\mathrm{T}_{c}$. This clearly indicates that the observed wavelength shift is a result of $\mathrm{H}$ desorption. 
As mentioned earlier, Si dangling bond are created due to the loss of $\mathrm{H}$. As a consequence, the propagation loss is expected to increase due to absorption. Despite this, we did not observe a considerable change in the propagation loss up to $400^{\circ} \mathrm{C}$ (Fig. 4). This suggests a reorganizing mechanism ${ }^{8}$ in the network below $400^{\circ} \mathrm{C}$, which reduces the dangling bond density. Beyond $400^{\circ} \mathrm{C}$ loss of most $\mathrm{H}$ atoms (Fig. 6 and 7) results in a high dangling bond density and a very high propagation loss.

The critical temperature $\left(\mathrm{T}_{c}\right)$ clearly demarks two regions; a chemically stable $\left(<\mathrm{T}_{c}\right)$ and an unstable $\left(>\mathrm{T}_{c}\right)$ operating region. These two regions can be used to carry out a postfabrication compensation of fabrication inaccuracies. It is clear from our experiments that above $\mathrm{T}_{c}$ the devices can be trimmed towards lower wavelength. On the other hand, since the material is chemically stable below $\mathrm{T}_{c}$, in this region the thermo-optic effect can be exploited to tune the device towards higher wavelengths. Due to the positive TO coefficient ${ }^{12}$, we observe a red shift of $90 \mathrm{pm} /{ }^{\circ} \mathrm{C}(d \lambda / d T)$. This is illustrated in Fig. 8, which shows the wavelength shift of a MZI as function of the operating temperature. The total achievable tuning then ranges from -6.6 to $+18 \mathrm{~nm}$.

In conclusion, we have studied the effect of a thermal treatment on PECVD depostited a-Si:H films by characterising photonic integrated devices. We have identified a critical temperature, which demarks two temperature ranges allowing for non-permanent (tuning) respectively permanent (trimming) changing of the material properties. By annealing asfabricated devices, we achieved trimming of wavelength selective photonic devices upto 6.6nm. In this case, the wavelength response was blue shifted. Despite this change, the propagation loss of the photonic wire waveguides did not change more then $0.2 \mathrm{~dB} / \mathrm{cm}$. Through FTIR spectroscopy, we showed that this trimming effect is a consequence of hydrogen loss during annealing. In addition, by using the TO effect, we demonstrated thermal 
tuning of $90 \mathrm{pm} /{ }^{\circ} \mathrm{C}$. We believe that in combination, trimming and tuning can be used to solve fabrication inaccuracies ${ }^{9}$ in $\mathrm{Si}$ photonic devices fabricated in a-Si:H films.

This work was partly supported by the European Union through the ICT-WADIMOS project and through the Dutch Smartmix program Memphis. 
* Electronic address: shankar@intec.ugent.be

[1] S. K. Selvaraja, E. Sleeckx, M. Schaekers, W. Bogaerts, D. Van Thourhout, P. Dumon, and R. Baets, Opt. Commun. 282, 1767 (2009).

[2] R. S. D. K. Sparacin, A. M. Agarwal, M. A. Beals, J. Michel, and L. C. Kimerling, Group IV photonics 2006, p.255.

[3] J. M. Pearce, J. Deng, R. W. Collins, and C. R. Wronski, Appl. Phys. Lett. 83, 3725 (2003).

[4] D. Han, J. Baugh, G. Yue, Q. Wang, Phys. Rev. B 62, 7169 (2000).

[5] W. Beyer and H. Wagner, J. Phys. 42, 783 (1981).

[6] T. Sakka, K. Toyoda and M. Iwasaki, Appl. Phys. Lett. 55, 1068 (1989).

[7] W. C. Hsiao, C. P. Liu, and Y. L. Wang, J. Phys. Chem. Solids, 69, 648-652 (2008).

[8] H. M. Branz and E. Iwaniczko, Phys. Rev. B 48, 17114 (1993).

[9] S. K. Selvaraja, W. Bogaerts, P. Dumon, D. Van Thourhout and R. Baets, IEEE J. Sel. Top. Quantum Electron. 16, 316 (2010).

[10] S. K. Selvaraja, P. Jaenen, W. Bogaerts, D. Van Thourhout, P. Dumon, and R. G. Baets, IEEE J. Lightwave Technol. 27, 4076 (2009).

[11] D. Taillaert, P. Bienstman, and R. Baets, Opt. Lett. 29, 2749 (2004).

[12] F. G. Della Corte, M. E. Montefusco, L. Moretti, I. Rendina and A. Rubino, Appl. Phys. Lett., 79, 168 (2001). 


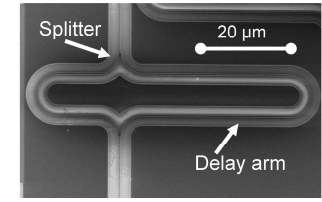

(a)

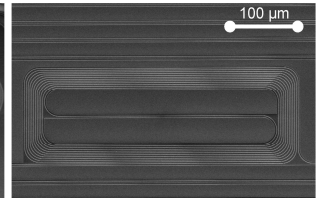

(b)

FIG. 1: One of the fabricated mach-zehnder interferometer and spiral photonic wire waveguide.

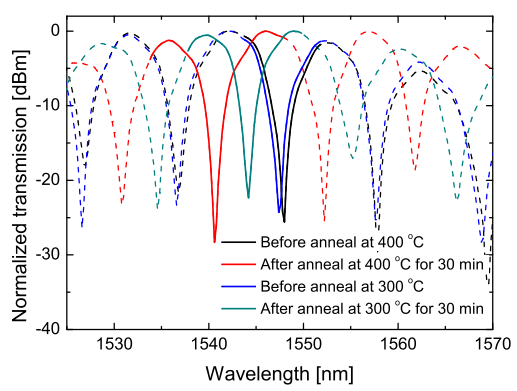

FIG. 2: (Color online) Transmission spectrum of MZI before and after annealing at 300 and 400

${ }^{o} \mathrm{C}$. The continuous lines show one free spectral range for clarity.

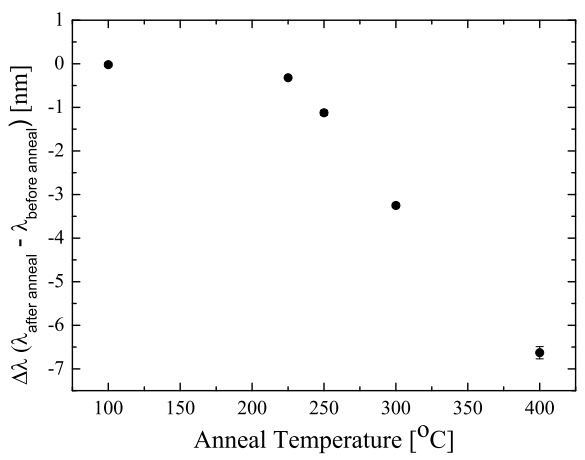

FIG. 3: Effect of anneal temperature on spectral shift of Mach-Zhender interferometer. 


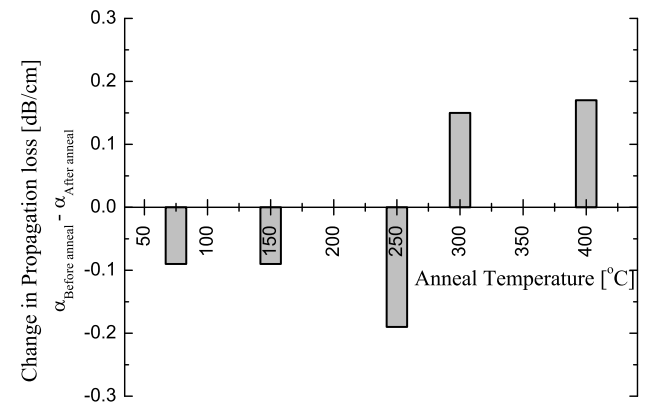

FIG. 4: Change in propagation loss of photonic wires as a function of anneal temperature.

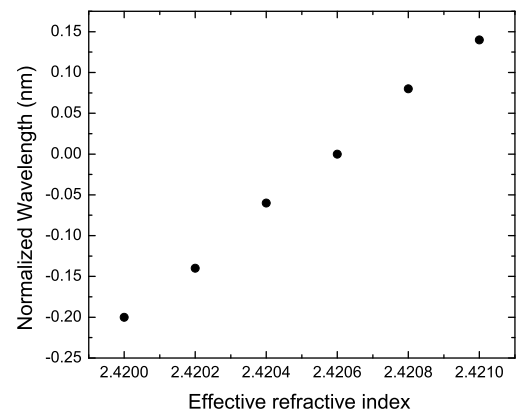

FIG. 5: Simulated spectral shift of a MZI as a function of effective refractive index of the photonice wire.

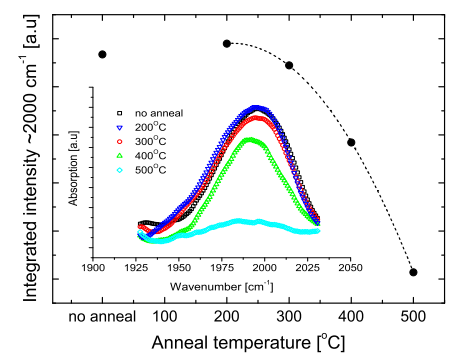

FIG. 6: (Color online) $(\bullet)$ FTIR spectrum at $2000 \mathrm{~cm}^{-1}$. 


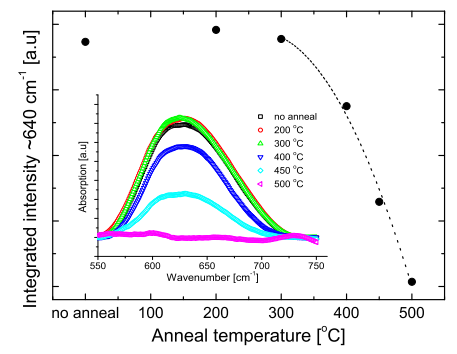

FIG. 7: (Color online)(•)FTIR spectrum at $640 \mathrm{~cm}^{-1}$.

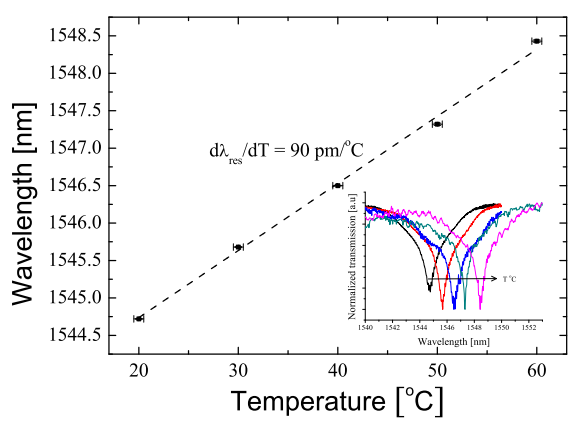

FIG. 8: (Color online) Thermo-optic effect of a-Si:H, inset shows the spectral shift of a MZI at different temperatures. 